\title{
Using a projected trompe l'oeil to highlight a church interior from the outside
}

\author{
A. Hoeben \\ fieldOfView \\ Lange Nieuwstraat 23b1 \\ 3111AC Schiedam \\ The Netherlands \\ aldo@fieldofview.com
}

\begin{abstract}
The St. Willibrordus Church in the city centre of Utrecht (The Netherlands) is a prime example of the Gothic Revival architecture. The richly decorated church interior is, unfortunately, one of the best kept secrets of the city. In the semi-permanent art installation presented in this paper, imagery showing the church interior is projected on the outside walls of the entrance of the church. By projecting onto a spherical mirror suspended from the entrance ceiling, the projection is reflected onto three walls and the ceiling. The resulting image, when seen from the street-level outside, creates a perspective illusion. This paper shows how the installation was implemented in the church in an unobtrusive way, and explains the calibration system that was developed for determining the pre-distortions required to project the Trompe L'Oeil.
\end{abstract}

Church interior. Art installation. Digital projection. Perspective manipulation. Optical illusion.

\section{INTRODUCTION}

In late 2007, the council of the city of Utrecht embarked on a project to create a new attraction for its historic city centre, aiming to keep some of the shopping public around during the evening hours.

A number of semi-permanent light art installations and sculptures was commissioned from different artists, each highlighting a different historical building or one of the many canal bridges in the city. The installations will all remain in place for a minimum of 10 years. The light art installations together constitute a walking route through the city centre under the name 'Trajectum Lumen'. The Trajectum Lumen was officially launched on 7 April 2010, and will have its climax in 2013 when the city of Utrecht celebrates the $300^{\text {th }}$ anniversary of the Treaty of Utrecht.

\subsection{St. Willibrord Church}

The St. Willibrord Church was built from 1874 to 1877 , and is a prime example of the Gothic Revival architecture of the time. When it was built the church was already boxed in by residential buildings. As a result, the church building is hidden

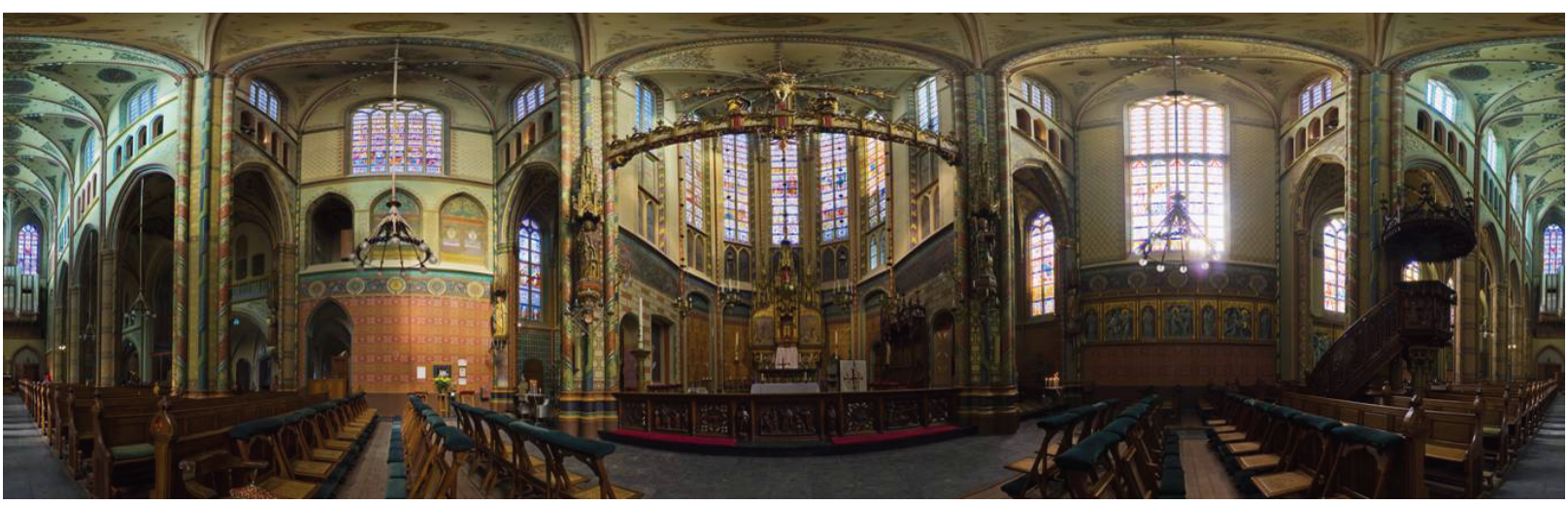

Figure 1: 360 degree panoramic view of the richly decorated St. Willibrord church interior 
from view when walking the dense streets of the historic city centre; it is very easy to miss the church unless you know what you are looking for. This is especially unfortunate because the richly decorated interior of the church (Figure 1) really sets the church apart from some of the more wellknown churches in Utrecht.

The church is in active service with a daily Latin mass and Sunday services. It is also used frequently as a venue for choir, organ and other classical music performances. In the summer, the church is open for the public after the morning mass until 4 o'clock.

The assignment brief focused on drawing attention to the church and its spectacular interior during the evening hours in a respectful manner, taking into account the monument status of the church and its active use, all within a limited budget. The light art installation was to require as few changes and additions to the building as possible, and all of them fully reversible. On the other hand the installation had to be durable enough to last at least 10 years.

\section{TROMPE L'OEIL INSTALLATION}

It was decided to show scenes and elements from the interior of the church projected on to the inside of the church porch. This church porch is on the walking route of the Trajectum Lumen, and is one of the few parts of the church actually visible from the street and recognisable as part of a church building (Figure 2).
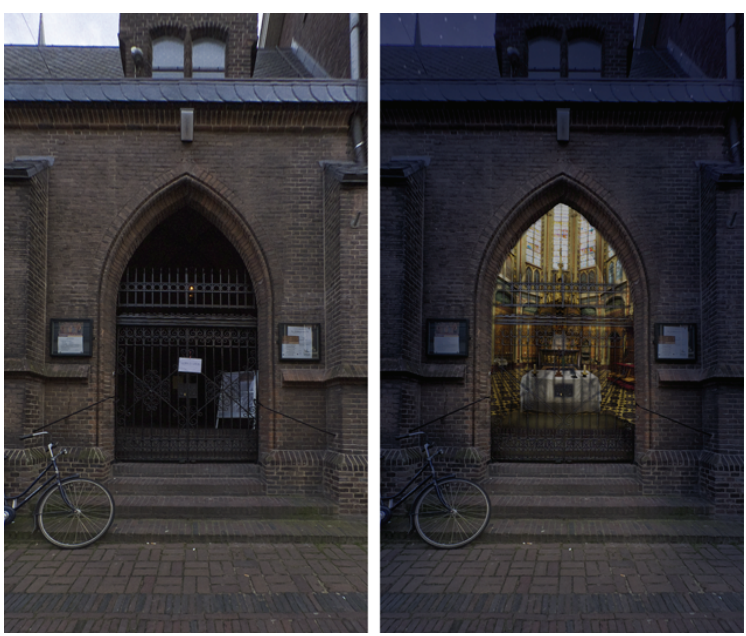

Figure 2: Church gate by day (left) and visualisation of the intended effect (right)

The porch has a gate, which is normally closed in the evening, and three doorways, two of which lead to the chapel and the church itself (the third doorway being a fake door). Above the main door, there is a small window which was likely used to light the entrance. This lighting feature has long since been replaced by a lamp suspended from the arched porch ceiling.

The small window allows for a projector to be inside the short hallway that leads to the actual church, shining out into the porch. The existing porch lighting was replaced by a $50 \mathrm{~cm}$ diameter stainless steel spherical mirror lampshade. The imagery is projected onto this spherical mirror and from there on reflects to the three walls and ceiling of the porch visible for people standing on the street in front of the church gate (Figure 3 ). The spherical mirror lamp shade still provides the functionality of lighting the porch when needed. In this way the required changes to the church, both functionally and structurally, have been kept to a minimum while keeping the delicate equipment inside, safe from most climate influences and vandalism.

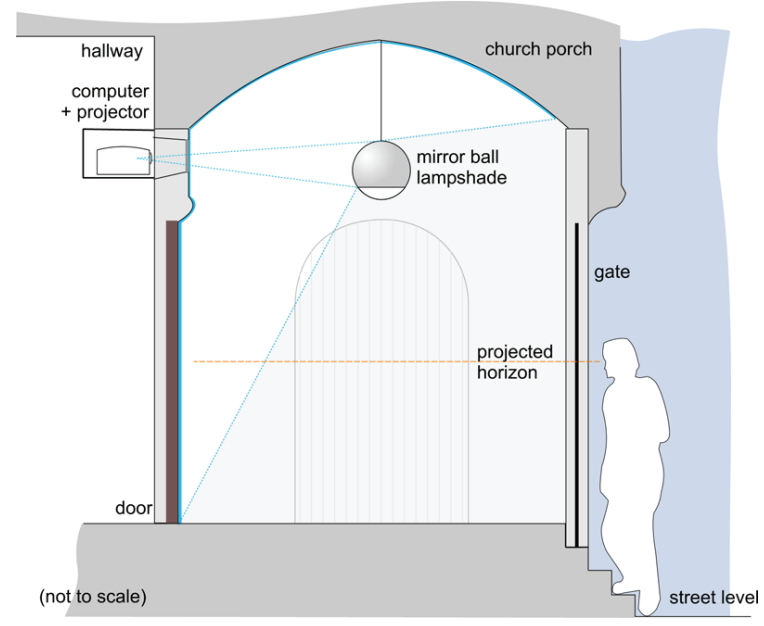

Figure 3: Schematic view of the Trompe L'Oeil installation

In order to support the suspension of disbelief among the audience, it was decided to project dynamic imagery, though care was taken not to disorient the spectators with overly dynamic camera moves. Subtle motion in the projected image makes it easier to separate visually the moving projection from the static wall structures.

\subsection{Related work}

In the past few years, augmenting non-planar surfaces with projected images has become a hit phenomenon. Valbuena (2007) was among the first to move from projecting on to purpose-built geometric structures to existing architecture. In most cases, this involves reconstructing a precise 3D model of the - often simple - geometry to be projected onto, manipulating this 3D geometry and its lighting and projecting specifically rendered 
views to achieve the desired optical illusion or other effect.

The geometry of the church porch is comparatively complex (Figure 4); The Gothic Revival architecture of the building adds a lot of geometric detail, including not-quite-symmetric arches, ornaments and the three monumental doors. Additionally, the different colours of aged brick wall and the three wooden doors provide a very unevenly coloured surface on which to project. Bimber et al. (2005) show how to use a Projector Camera System to compensate for uneven surface colour and reflectance properties, as well as geometric disturbances without prior knowledge of the projection surface.

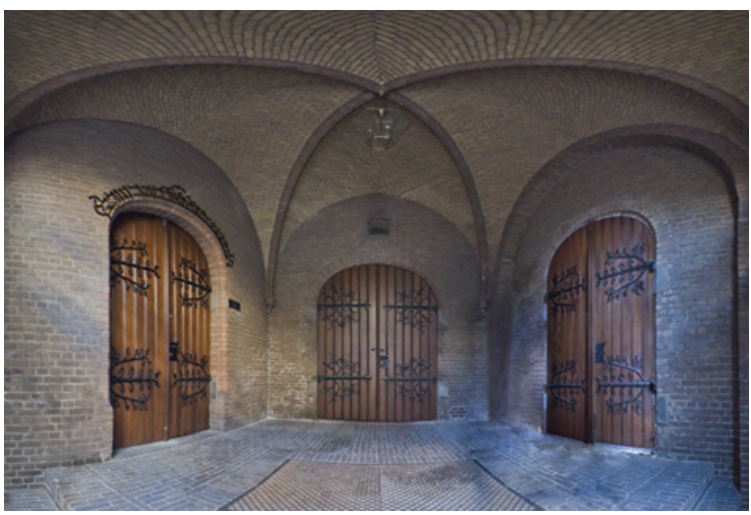

Figure 4: Cropped equirectangular view of the church porch

Methods suggested to use multiple projectors to increase the field of view of the projection (Surati, 1999) are not suitable for this project due to budgetary and space constraints. Bourke (2005) first explored using a single commodity projector and spherical mirrors to obtain a high field of view from a single projection.

\subsection{Projection calibration}

To project a convincing wide-angle optical illusion on to the walls and ceiling of the porch, a specifically distorted image must be projected on to the spherical mirror. The wide-angle content is prepared for pre-distortion as a video with a specific perspective. In this case, a cropped 180 degree equirectangular projection was chosen.

The calibration method starts by projecting a number of Gray-coded binary calibration patterns (Salvi et al., 2004) on to the spherical mirror with the mirror and projector both securely placed on their final location. The XGA resolution of the projector dictates that a total of 20 calibration patterns are required to uniquely identify each pixel in the projectable image; 10 each for the horizontal and vertical position of the pixels. The resulting projection is photographed using a 180 degree fisheye lens from the intended position of the audience at the gate of the church porch (Figure 5). These calibration images are then remapped to the same perspective and dimensions as the equirectangular source video (Figure 6).
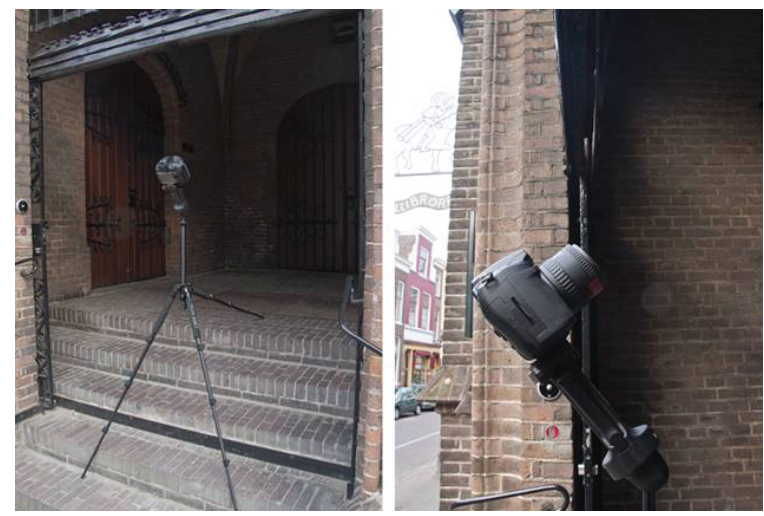

Figure 5: Calibration-pattern photography from the audience' viewpoint

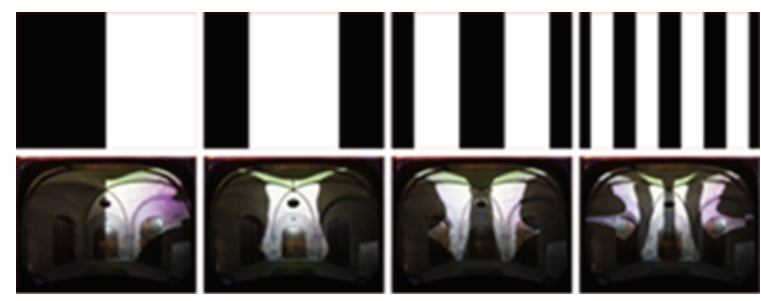

Figure 6: First 4 Gray coded binary patterns (top) and corresponding photographed calibration photographs

For each pixel in the combined equirectangular calibration photographs, the originating projected pixel can be recovered simply by converting the Gray-coded pattern back into a natural binary code. This yields a 10 bit horizontal and vertical coordinate. For convenience the recovered coordinate map is stored as a 16 bit RGB image, where the red channel represents the horizontal coordinate, the green channel represents the vertical coordinate and the blue channel is left unused. This image can then be processed in an image editor such as Adobe Photoshop to remove noise and artefacts.

One of the artefacts present in the coordinate-map is indirect light in the 'shadow areas' where the projection does not reach. Due to the geometry of the porch, there are certain areas that are not visible from the standpoint of the projection, but they are visible from the standpoint of the audience. Ideally, these shadows would show up as black in each of the calibration photos, but due to ambient reflections these areas show up as noise in the coordinate map. These noise areas are removed manually from the coordinate map before continuing. 


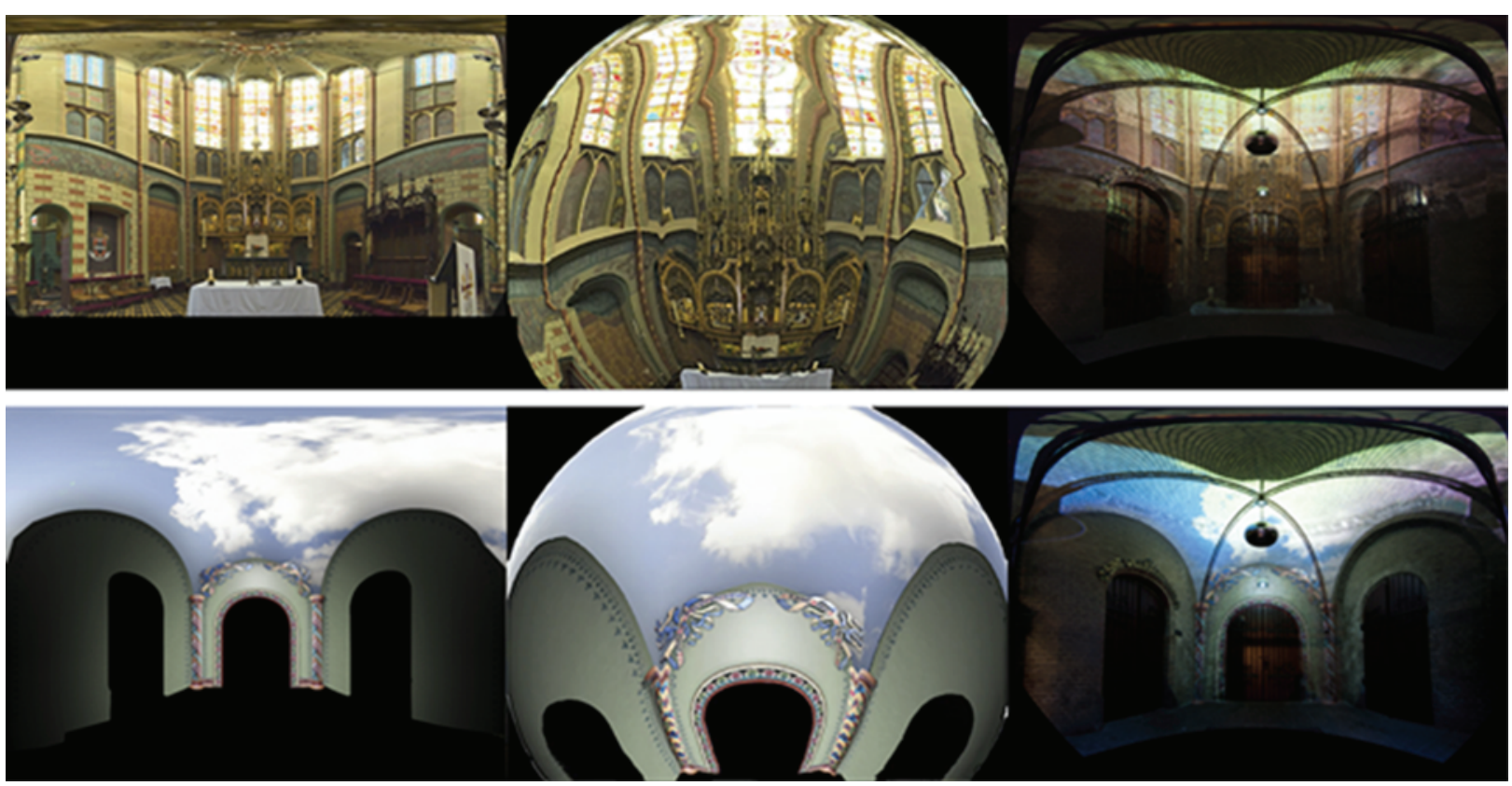

Figure 7: Two frames of the projected content. Source in cropped equirectangular perspective (left), pre-distorted image for projection (centre) and resulting projected image in equirectangular perspective (right)

The coordinate map contains the projection pixel for each of the 'spots' on the wall. Because the calibration photographs have the same projection as the prepared source video, the coordinate map can also be used to link a pixel in the source panorama imagery to a pixel in the projection. The next step in the calibration is to create an inverse of the coordinate map. This results in a new coordinate map that links each pixel in the projected image to a part of the wall, and to a part of the source panorama image.

Creating the inverse coordinate map is done by traversing all pixels in the original coordinate map and setting the pixels at the recovered coordinate in the new map to the coordinate of the current pixel. This creates a new map that is again stored as a 16 bit RGB image for convenience. This map is also processed in an image editor to fill holes caused by shadows, as well as remove noise.

Finally the cleaned-up inverse coordinate-map is used to distort the map to create the pre-distorted projection image from the source panorama video. The resulting image, when projected onto the spherical mirror fits perfectly to the geometry, and is geometrically corrected for the audience viewpoint (Figure 7).

Though the pre-distortion of the source video can be carried out in real-time using current mainstream GPU processing, it was decided to precompute the distortion of the video to save a little computing power. Even if the savings are small compared to the power consumption of the projector, it will add up to a substantial saving over the period of ten years.

To enhance the projection further it is possible to compensate for the colour and other properties of the wall on to which each pixel is projected, as Bimber (2005) demonstrated. To do this we can use the same inverse coordinate map on a photograph of the walls and doors taken from the same location. Because of the homography of light, the resulting image is like a photo taken through the lens of the projector (Sen, 2005). This image shows the colour and brightness of the surface where each of the projection pixels will be projected.

During the execution of the installation, one of the main issues was how even the smallest movement of the spherical mirror affected the projection. At first the spherical mirror was suspended from the ceiling by its power cord, leaving it to sway in the wind by up to a couple of millimetres. This had a dramatic impact on the reflected projection. By making a stiff connection from the ceiling to the lamp, the effects of draught and wind were greatly reduced. Unfortunately, a fully rigid connection to the ceiling could not be made due to the status of the building, so the spherical mirror can still move slightly over time. The motion of the mirror is not so dramatic that the illusion breaks; if there is a very strong wind outside, chances of having an audience are slim anyway. However, because a tiny displacement of the mirror causes the compensation of the background surface to break dramatically (Bimber, 2005), it was decided not to compensate the projection for background brightness. 

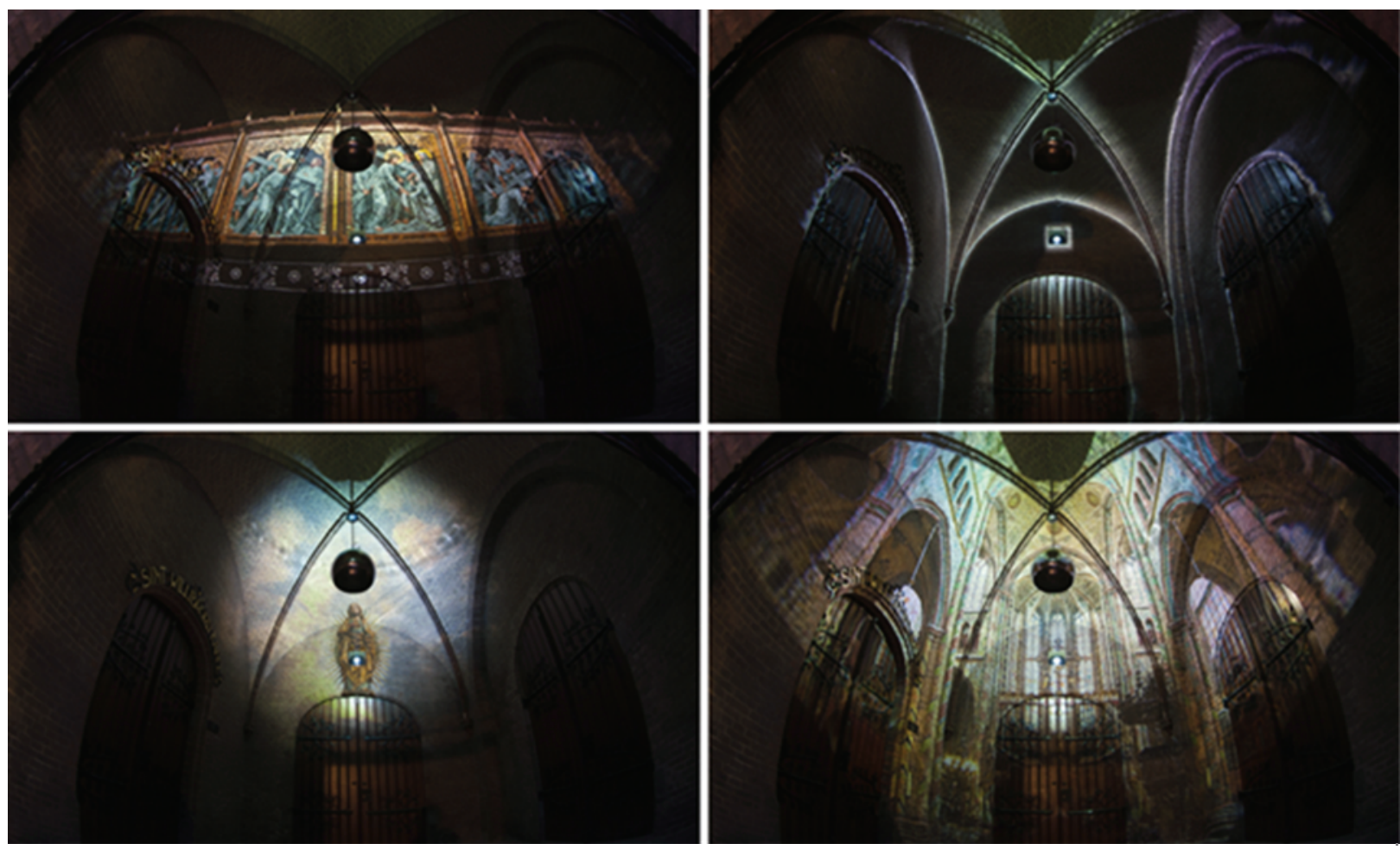

Figure 8: Additional photographs of the projected result of the Trompe L'Oeil installation

\section{RESULTS}

The Trompe L'Oeil was launched on 1 October 2009 , and is showing daily from sunset through midnight. Since then, the installation already turned heads of occasional passers-by. On 7 April 2010, the full Trajectum Lumen route is opened for the public, and since then it has been attracting an additional audience looking specifically for the church and the Trompe L'Oeil installation. In this regard, the installation has certainly fulfilled its main goal of drawing attention to the church in a positive manner.

The method of calibrating the reflected projection for arbitrary geometry has proven successful even for extremely wide field of view projections using a curved mirror (Figure 8). The stainless steel mirror that was manufactured for the installation has some minor dents in it due to the manufacturing method. These lead to distortions in the reflection. But fortunately these additional distortions are mostly removed in the calibration process. The interesting observation is that a camera projector system can reduce effects of not only sub-optimal projection surfaces but probably also lower-precision, lowercost optics in the projection path.

Since the project completion, the same projection and calibration techniques have been successfully applied to create a dual projector, 360 degree projection in an art gallery, and work is ongoing to streamline the workflow.

\section{ACKNOWLEDGEMENTS}

The author would like to thank the city of Utrecht for the opportunity to do this project, and for the freedom given during its execution. Jeroen Degenaar of Do Not X-Ray has been instrumental to the project for his advice on projectors and lenses to use.

\section{REFERENCES}

Bimber, O., Emmerling, A., and Klemmer, T. (2005) Embedded entertainment with smart projectors. IEEE Computer (cover feature), pp. 56-63, January 2005.

Bourke, P. (2005) Spherical mirror (Mirrordome): A new approach to hemispherical dome projection. Planetarian, 34:4, pp 6-9, December 2005.

Salvi, J., Pagés, J., and Batlle, J. (2004) Pattern codification strategies in structured light systems. Pattern Recognition, 37:4, pp. 827-849, April 2004.

Sen, P., Chen, B., Garg, G., Marschner, S. R., Horowitz, M., Levoy M., and Lensch, H. P. A. (2005) Dual photography. ACM Transactions on Graphics, 24:3, July 2005. 
Surati, R. (1999) A Scalable Self-Calibrating

Technology for Seamless Large-Scale Displays.

$\mathrm{PhD}$ thesis, Department of Electrical Engineering

and Computer Science, Massachussetts Institute of

Technology, USA, 1999.
Valbuena, P. (2007) pablo valbuena.

http://pablovalbuena.com (2 April 2010). 